Int. J. Dev. Biol. 49: 409-416 (2005)

doi: $10.1387 / \mathrm{ijdb} .041909$ sd

Original Article

\title{
Chromosomal disorders and nuclear and cell destruction in cleaving human embryos
}

\author{
STEFKA M. DELIMITREVA*,1, RALITSA S. ZHIVKOVA ${ }^{1}$, ILYA T.S. VATEV ${ }^{1}$ and DRAGA I. TONCHEVA ${ }^{2}$ \\ ${ }^{1}$ Human IVF center, Department of Biology and ${ }^{2}$ Department of Medical Genetics, Medical University, Sofia, Bulgaria
}

\begin{abstract}
Three types of defects of preimplantation embryogenesis contribute to the developmental arrest of cleaving human embryos: blastomere fragmentation, abnormal nuclear status and chromosomal disorders. Data concerning the relation and succession of these abnormalities during first mitotic cycles of the human zygote are controversial and mainly empirical at present. In this study we have performed simultaneous evaluation of blastomere fragmentation, nuclear apoptotic changes and the ploidy of four chromosomes $(1,5,19$ and $X$ or 18, 21, $X$ and $Y)$ in 193 human embryos. Another group of 28 embryos was subjected to TUNEL for confirmation of apoptosis in blastomere nuclei. Nuclei with apoptotic chromatin were seen in nearly 1/10 of blastomeres of embryos with good morphology and in more than 1/5 of blastomeres of embryos with more than $20 \%$ fragmentation. The correct number of investigated chromosomes was registered in $85.2 \%$ of successfully tested embryos. Chromatin apoptotic changes are the only limiting factor for the success of chromosomal FISH tests. Nearly 1/2 of embryos with at least one apoptotic nucleus were chromosomally abnormal. For the embryos that contain only normal nuclei, the rate of ploid normality was more than $89 \%$. The rate of euploidy was higher $(66 \%)$ in embryos with a significant degree of cell fragmentation. Moderate cell fragmentation was not related to significant increase of chromatin and chromosomal disorders. In a substantial portion of abnormal blastomeres, chromatin damage preceded cell fragmentation. Nuclear destruction in human blastomeres was illustrated by fluorograms of different stages of chromatin lesions.
\end{abstract}

KEY WORDS: preimplantation, aneuploidy, apoptosis, blastomere fragmentation, in vitro fertilization

\section{Introduction}

The success rate of assisted reproductive techniques in human is relatively low. Despite the efforts to improve the conditions of gamete and embryo culture, implantation rate per embryo rarely exceeds $15 \%$ (Hardy et al., 2002). This suggests that the low implantation rate could not be a result only of the procedure or in vitro conditions, but real features of oogenesis and early embryogenesis are involved. These features concern both in vitro and in vivo conceptions and contribute to the low human fertility (Buster et al., 1985, Handyside, 1996).

Criteria for embryo selection before intrauterine transfer are mainly morphological characteristics and, in a limited number of IVF (in vitro fertilization) centers, chromosomal normality. Main morphological scores are: adequate cell number and volume; the degree and pattern of blastomere fragmentation; presence of multinucleated and anucleated blastomeres. Preimplantation genetic tests are performed only in special cases - parental chromosomal defects or sex related diseases, advanced maternal age, recurrent miscarriages or multiple unsuccessful IVF attempts (Munne, 2003).

Chromosomal investigations of cleavage stage embryos show that ploid errors could be one of the main causes of low implantation rate of human embryos (Munne, 2003, Delhanty, 2001). There are data (Evsikov and Verlinsky, 1998, Fulka et al., 1999, Hunt and Hassold, 2002) that chromosomal abnormalities are consequence of low or absent efficiency of cell cycle checkpoints during the final steps of female meiosis and first mitoses of the zygote (male meiotic checkpoints are fare more effective). Cell cycle errors result in unexpectedly high incidence of ploid abnormalities in mature oocytes and apparently normal cleaving embryos (Evsikov and Verlinsky, 1998, Fulka et al., 1999). Investigations of the whole chromosomal set reveal that only about $25 \%$ of cleavage stage human embryos contain only chromosomally normal cells (Wells and Delhanty, 2000). There is no strict

Abbreviations used in this paper: FISH, fluorescent in situ hybridization; IVF, in vitro fertilization. 
correlation between morphological and ploid abnormalities. Chromosomal errors occur in all morphological classes, but are observed more frequently in fragmented and arrested embryos (Delhanty, 2001, Coonen et al., 1994, Magli ei al., 2000, Munne et al., 1995, Pellestor et al., 1994). In the last years several working groups investigate the defects of nuclear number and morphology. They register abnormal number of nuclear structures in approximately $1 / 3$ of cleaving embryos (Balakier and Cadesky, 2004, Van Royen et al., 2003). Interphase blastomere nuclei can be classified as follows: mononucleated, mononucleated with micronuclei, binucleated, multinucleated, apoptotic (Chatzimeletiou etal., 2005). Binucleated blastomeres contain two equal- or rarely unequal-sized nuclei, most often with normal chromatin appearance. Probably this kind of multinucleated blastomeres occur after normal karyokinesis without subsequent cytokinesis, i. e. they could be referred to the cell cycle faults (Hardy et al., 1993, Pickering et al., 1995). In these cases no increase of the rate of chromosomal errors is observed (Kligman et al., 1996). In the multinucleated blastomeres (containing more than two relatively small nuclei) the rate of chromosomal abnormalities is very high. Multiple small nuclei and micronuclei could arise by abnormal organisation of the mitotic spindle, anaphase lagging or nuclear fragmentation. This could be a manifestation of an apoptotic process in a dying cell. This idea is supported by the fact that nuclear apoptotic changes are more common in fragmented or arrested embryos, i. e. embryos with poor developmental prognosis (Jurisicova et al., 1996, Warner et al., 1998). According to other authors, blastomere fragmentation is not a result but a cause of nuclear apoptosis or these two processes are not related (Antczak and Van Blerkom, 1999, Martinez et al., 2002, Van Blerkom et al., 2001). For instance, multiple nuclei could arise by abnormal packing of the chromatin after mitotic division (Kligman et al., 1996). Although it is clear that blastomere fragmentation, cell cycle errors and apoptosis decrease embryo viability and pregnancy rate, the data about relation and succession of these phenomena are empirical and controversial at present. In an earlier study, we have scored abnormal chromatin distribution (nuclear fragmentation and condensation) in human embryos with good morphology (Delimitreva, 2002). Chromatin abnormalities were observed in a substantial portion of embryos with no or minimal blastomere fragmentation. These results suggest that nuclear lesions precede cell destruction and are rather a cause than a consequence of cell fragmentation.

In the present study, we have investigated the relation between the following embryonic characteristics: the degree of blastomere fragmentation, apoptotic chromatin lesions and chromosomal status. The results derived from embryos donated for research after successful and unsuccessful IVF cycles were compared.

\section{Results}

\section{Morphology}

A total of 108 embryos were donated after successful and 103 after unsuccessful IFV cycles. Prior to fixation, all 211 embryos (stage 3-10 cells) were classified according to their blastomere fragmentation. Proportions of morphological classes $A, B$ and $C$ were relatively equal - 69 embryos (33\%) in class A, $79(37 \%)$ in class $\mathrm{B}$ and $63(30 \%)$ in class $\mathrm{C}$.
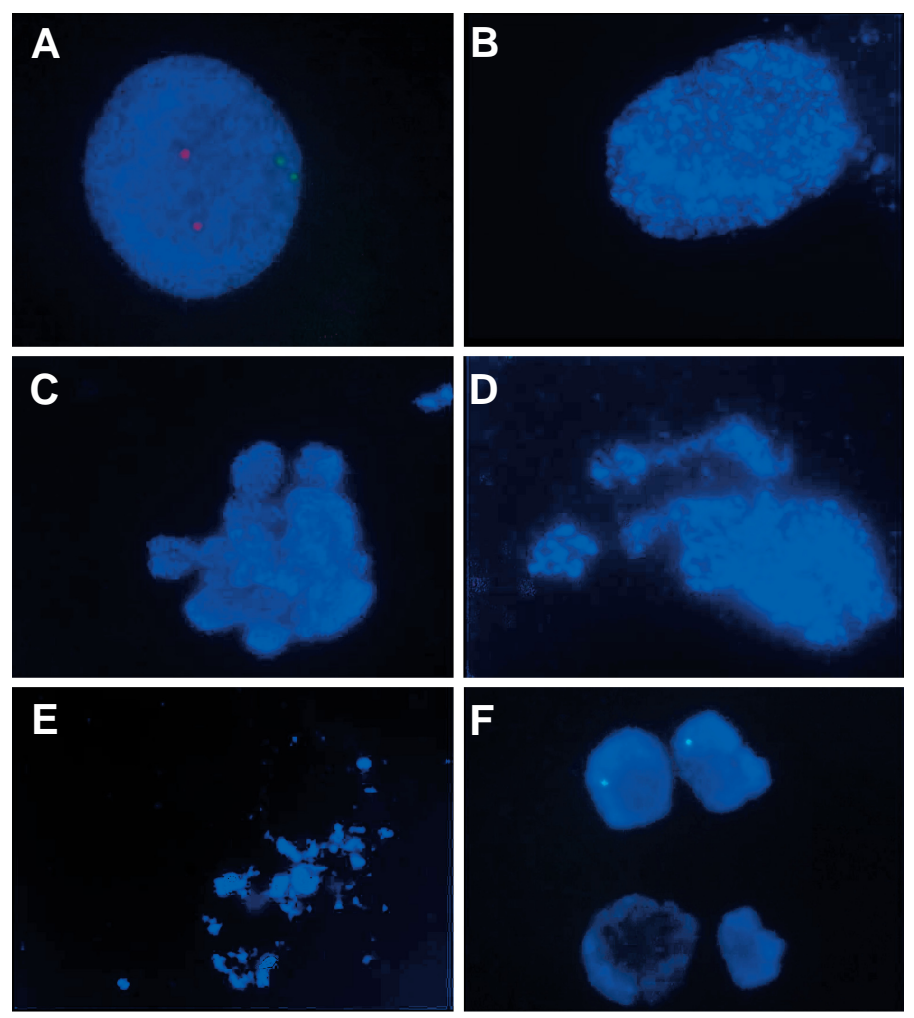

Fig. 1. Fluorescent image of blastomere chromatin. (A) A normal nucleus with normal FISH for chromosomes 18 (two spots at the periphery) and 21 (two spots in the center). (B,C,D,E) Subsequent stages of chromatin condensation and fragmentation. (F) Nuclear fragments with FISH for chromosome 18 and missing locus-specific signals for chromosome 21. The nuclei were counterstained with DAPI.

\section{Chromatin status}

One hundred and eighty-three embryos were prepared for simultaneous evaluation of chromatin status (by fluorescent reaction of the chromatin counterstaining) and chromosomal normality (by FISH). Ninety-four embryos in this group were from women who achieved pregnancy and 89 from non-pregnant women. Total number of fixed blastomeres was 919 . In 46 of them, neither nucleus nor clear nuclear remains were observed -28 necrotic cells and 18 enucleated blastomere fragments. Chromatin status of non-fragmented nuclei with pale even fluorescent reaction or mitotic chromosomal sets was defined as normal. Fragmented, flocculent or contracted nuclei and nuclei with heterogeneous chromatin consistence demonstrating clusters with bright reaction, were defined as abnormal. Discrepancy between phase contrast observations before blastomere fixation and fluorescent images was noticed. Fluorograms of normally and abnormally looking nuclei are presented at Fig 1. Fig. 1 A shows a nucleus with normal chromatin appearance and normal number of chromosomes 18 and 12 (green and red respectively). The images 1B, $1 \mathrm{C}, 1 \mathrm{D}$ and $1 \mathrm{E}$ are arranged according to the presumable chronology of chromatin fragmentation and condensation. Some of the abnormal images were derived from blastomeres described prior to fixation as normal and mononucleated (1B and $1 \mathrm{C}$ ) and the rest - from blastomeres initially defined as multinucleated (1D) or enucleated (1E). 
A total of 873 nuclei were observed. In 742 (85\%) of them chromatin status was concerned normal and in 131 (15\%) abnormal. There was no difference between the proportions of abnormal nuclei from pregnant and non-pregnant women-15.3\% (71 out of 463 nuclei) and 14.6\% (60 from 410 ) respectively.

\section{Chromatin status / morphology}

The rate of nuclei with abnormal chromatin status (Table 1) was approximately equal in class $A$ and class $B$ embryos respectively $9.6 \%$ (28 abnormal out of 291 available nuclei) and $12.2 \%$ (34 abnormal from $\left.278,0.01<P \chi^{2}\right)<0.05$ ). The percentage of abnormal nuclei was significantly higher in class $\mathrm{C}$ embryos $22.7 \%$ (69 from 337 available nuclei, $\mathrm{P}\left(\chi^{2}\right)<0.001$ ).

\section{Chromatin status / mitotic rate}

Approximately $19 \%$ (34) of embryos had at least one blastomere with abnormal nucleus. This proportion was higher in embryos at advanced cleavage stage (Fig. 2). Before the start of third mitotic round, at 3 - and 4-cell stage, $12 \%$ of observed nuclei (36 from 302) were abnormal and 18\% of embryos (16 from 87 ) had at least one abnormal nucleus. Before completion of third mitotic round (5- to 8 -cell stage), $15 \%$ of nuclei $(79 / 502)$ were abnormal and $15 \%$ of embryos (13/88) had abnormal nuclei. We noticed that the percentage of abnormal nuclei was significantly higher $\left(\mathrm{P}\left(\chi^{2}\right)<0.001\right)$ in embryos with completed third mitotic round (with more than 8 cells). Abnormal nuclei were $23 \%(16 / 69)$ distributed in $63 \%$ of embryos in this group (5/8).

\section{Ploid status}

Fifty-two embryos were tested for chromosomes 1, 5, 19 and $X$ and 131 - for chromosomes $18,21, X$ and $Y$. FISH reaction was scored only in normal nuclei. In cases with two normal nuclei in one blastomere, each nucleus was scored separately. According to the established criteria (Munne et al., 1998), fluorescent spots noticeably larger or smaller than other signals in the same nucleus were considered non-specific hybridization. Paired small signals and signals found in close vicinity to each other were counted as

\section{TABLE 1}

\section{CHROMATIN STATUS / MORPHOLOGY}

\begin{tabular}{lcccccc} 
& $\begin{array}{c}\text { No. of } \\
\text { embryos }\end{array}$ & $\begin{array}{c}\text { No. of fixed } \\
\text { blastomeres }\end{array}$ & Necrotic & Anucleated & $\begin{array}{c}\text { Normal } \\
\text { nuclei }\end{array}$ & $\begin{array}{c}\text { Abnormal } \\
\text { nuclei (\%) }\end{array}$ \\
\hline Class A & 61 & 295 & 0 & 4 & 263 & $28(9.6 \%)$ \\
Class B & 69 & 287 & 3 & 6 & 244 & $34(12.2 \%)$ \\
Class C & 53 & 337 & 25 & 8 & 235 & $69(22.7 \%)$ \\
Total & 183 & 919 & 28 & 18 & 742 & $131(15.0 \%)$ \\
\hline
\end{tabular}

TABLE 2

\section{PLOID STATUS / MORPHOLOGY}

\begin{tabular}{lcccccc} 
& $\begin{array}{c}\text { Normal embryos } \\
(\%)\end{array}$ & & \multicolumn{5}{c}{$\begin{array}{c}\text { Abnormal embryos } \\
\text { triploid }\end{array}$} & $\begin{array}{cccccc}\text { aneuploid } \\
\text { Class A }\end{array}$ & $51(83.6 \%)$ & & 1 & 2 & 6 & 0 & 1 \\
Class B & $58(84.1 \%)$ & 1 & 1 & 5 & 2 & 2 \\
Class C & $35(66.0 \%)$ & 0 & 1 & 4 & 2 & 11 \\
Total & $144(78.7 \%)$ & 2 & 4 & 15 & 4 & 14 \\
\hline
\end{tabular}

*embryos with less than required number of normal nuclei

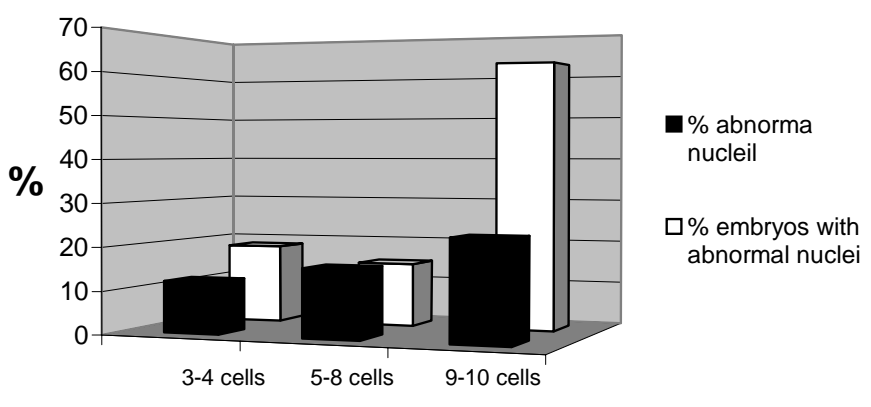

Fig. 2. Cleavage stage / chromatin status. Percentage of abnormal nuclei and embryos with at least one abnormal nucleus at different cleavage stages.

one, as they represented reaction for two sister chromatids. Successful FISH reaction was registered in practically all normal nuclei, except two fixed folded. In our earlier studies (Delimitreva, 2002, Delimitreva et al., 2001) we have noted that FISH reaction is not reliable in nuclei with any degree of chromatin condensation. FISH signals were observed in some fragmented and flocculent nuclei with relatively homogeneous chromatin, but the number of spots was chaotic and not useful as a result. Most often, some of locus-specific signals but not centromeric ones were lost in fragmented nuclei (Fig. 1F) This situation could be wrongly recognized as monosomy or nullisomy of a certain chromosome. Chromosome status of embryos was determined if at least two successfully hybridized nuclei were available for 3- and 4-cell stage embryos or at least three reacted nuclei were observed in embryos with more than 4 cells.

Ploid status of tested chromosomes was determined in 169 embryos. The rest 14 embryos did not have sufficient number of normal nuclei. Normal chromosome number was observed in 144 embryos (85.2\%). For the two groups of embryos the rate of ploid normality was very close - for chromosomes $1,5,19$ and $X$ this rate was $83 \%$, for chromosomes $18,21, X$ and $Y$ it was $86.1 \%$. Fifteen embryos $(8.9 \%)$ were mosaic, $4(2.4 \%)$ - aneuploid, 4 $(2.4 \%)$ - chaotic and $2(1.2 \%)$ embryos were triploid. There was no difference in the rate of chromosomal normality for pregnant and non-pregnant women - it was $86.2 \%$ (75 normal embryos from 87 successfully tested) and $84.8 \%$ ( 69 from 82 ) respectively. Comparison of morphological, ploid and chromatin status of embryos donated after successful and unsuccessful IVF cycles are summarized in Fig. 3.

\section{Ploid status / morphology}

The distribution of embryos with abnormal ploidy in classes A, $B$ and $C$ is presented in Table 2. There was no reliable difference between ploid normality of class $A$ and class $B$ embryos - $85 \%$ and $86.8 \%$ respectively, $P\left(\chi^{2}\right)>0.05$. For class $C$ this rate was slightly different -42 successfully tested, 35 of them were normal $(83,3 \%), 4$ mosaic, 1 aneuploid, 2 chaotic. The difference was more pronounced $\left(\mathrm{P}\left(\chi^{2}\right)<0.001\right)$ when chromatin abnormalities were included in the calculations. The rate of total normality was calculated as a relation of chromosomally normal embryos and all tested embryos, i. e. embryos with high proportion of fragmented and condensed nuclei (not defined by FISH) were recognized as abnormal. For class A it was $83.6 \%$ (51 from 61 ), for class B - 
$84.1 \%(58 / 69)$ and for class C - 66\% (35/53). The rates of nuclear and ploid normality of different morphological classes are compared in Fig. 4.

\section{Chromatin status / ploid status}

We have registered 25 chromosomally abnormal embryos. They were more frequent among the embryos with at least one abnormal nucleus. Abnormal nuclei were observed in 35 embryos, but only 20 of them had sufficient number of normal nuclei for evaluation of ploidy. Eleven of them were chromosomally normal (55\%), 2 - triploid, 3 - mosaic, 3 - chaotic and 1 aneuploid. The rest of observed embryos (194) had only normal nuclei, 133 of them were chromosomally normal (89.3\%), 12 mosaic, 3 aneuploid and 1 chaotic.

\section{TUNEL}

Twenty-eight embryos at 3-6-cell stage were fixed in paraformaldehyde and subjected to TUNEL. A total of 141 blastomeres were examined, 5 of them had necrotic appearance. Signs of advanced apoptosis (nuclear fragmentation and/or uneven chromatin condensation) were observed in 17 blastomeres $(12.5 \%)$. Apparently normal nuclei were seen in 119 blastomeres (87.5\%). Presence of DNA breakage, which is one of the signs of apoptotic process, was demonstrated by positive TUNEL-test in 8 of them (6.7\%, Fig. 5). The total rate of apoptosis was $18.4 \%$.

\section{TUNEL / morphology}

The rate of advanced nuclear destruction (chromatin condensation and/or fragmentation) differed in classes $\mathrm{A}, \mathrm{B}$ and $\mathrm{C}$ (Table 3 ). It was $10.1 \%$ for class $A, 8.3 \%$ for class $B$ and $17.6 \%$ for class C. The rate of early apoptotic changes in apparently good nuclei was $2.9 \%$ for class A ( 1 nucleus with normal chromatin and positive TUNEL / 34 normal nuclei), $6.8 \%$ for class B (3/44) and $9.8 \%$ for class $C(4 / 37)$. There was no significant difference between the data for class $A$ and $B$. For class $C$ data were noticeably different (embryo number was not sufficient to register

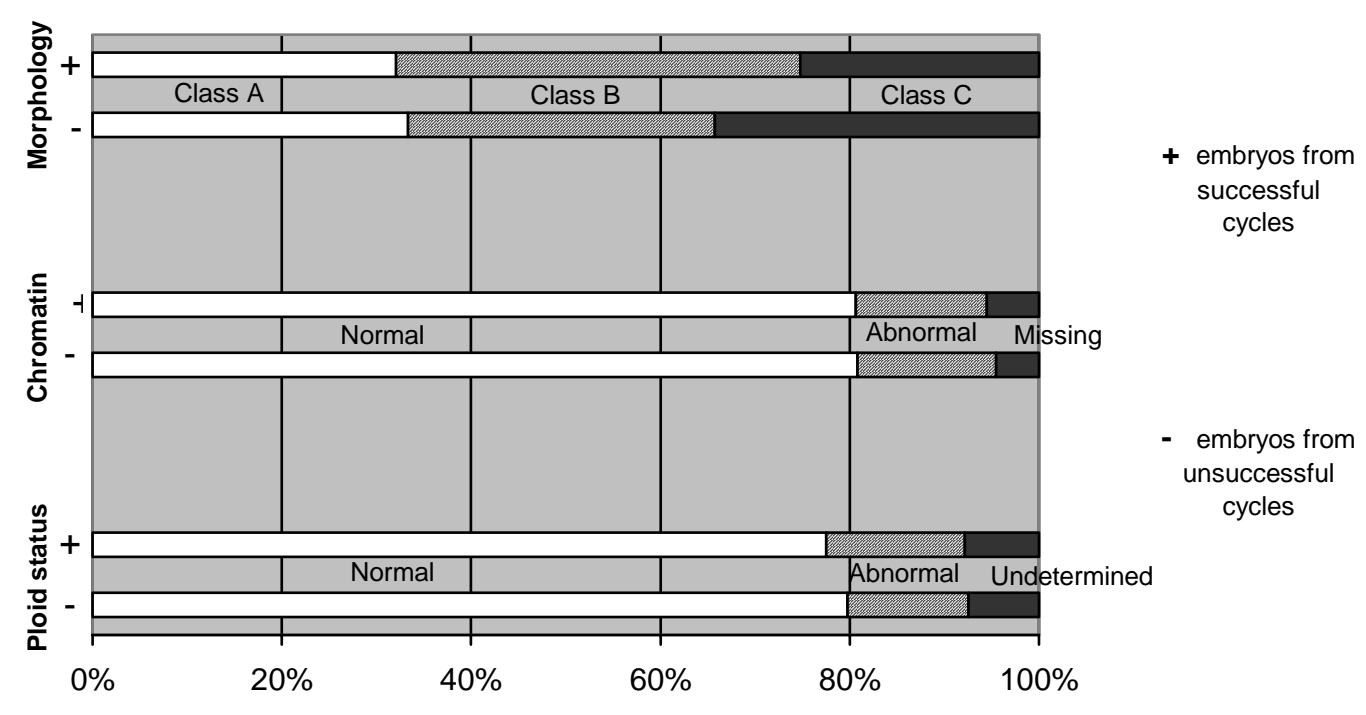

Fig. 3. Comparison of morphology, chromatin and ploid normality of supernumerary embryos from successful and unsuccessful IVF cycles.
TABLE 3

\begin{tabular}{|c|c|c|c|c|c|c|}
\hline & \multirow{2}{*}{$\begin{array}{c}\text { No. of } \\
\text { embryos }\end{array}$} & \multirow{2}{*}{$\begin{array}{l}\text { No. of } \\
\text { nuclei }\end{array}$} & \multicolumn{2}{|c|}{ Normal nuclei } & \multirow{2}{*}{$\begin{array}{l}\text { Abnormal } \\
\text { nuclei }\end{array}$} & \multirow{2}{*}{$\begin{array}{c}\text { Apoptotic rate } \\
\text { (\%) }\end{array}$} \\
\hline & & & - TUNEL & + TUNEL & & \\
\hline Class A & 8 & 38 & 33 & 1 & 4 & $5 / 38(13.2 \%)$ \\
\hline Class B & 10 & 48 & 41 & 3 & 4 & $7 / 48(14.6 \%)$ \\
\hline Class C & 10 & 50 & 37 & 4 & 9 & $13 / 50(26 \%)$ \\
\hline \multirow[t]{2}{*}{ Total } & 28 & 136 & 111 & 8 & 17 & $25 / 136(18.4 \%)$ \\
\hline & & & & Apop & uclei & \\
\hline
\end{tabular}

statistically reliable difference, $\left.\mathrm{P}\left(\chi^{2}\right)>0.05\right)$. The proportions of apoptotic nuclei registered by TUNEL and chromatin fluorescent reaction were very similar (Fig. 6).

\section{Discussion}

In published studies on multinucleated blastomeres, only the number of nuclear structures is commented. Our detailed observations of all fixed blastomeres suggest that it is necessary to specify the definition of blastomere multinucleation - chromatin defects and even advanced apoptotic changes can be observed in apparently normal mononucleated blastomeres; registered multinucleation could be a result either of nuclear fragmentation, or of failed cytokinesis after normal kariokinesis; some anucleated cells or big fragments are blastomeres with dying nuclei. Abnormal chromatin distribution, except for multinucleation, is not commented as a possible reason for failures of FISH tests in preimplantation chromosomal surveys. As far as we know, our study is the first that focuses on this question. Our modified airdrying fixation applied before FISH seems suitable for simultaneous estimation of nuclear destruction and ploidy. We have noticed that some simultaneously fixed neighbor nuclei had different rate of FISH success and this was always related to different rate of chromatin condensation. We assumed that in these cases, the absence of FISH signals was not caused by failed hybridization but by chromatin lesions.

There are classical morphological criteria for nuclear apoptosis, accepted even before the biochemical and genetic fenomena related to cell death were understood. Our images of abnormal nuclei were similar to the pictures of apoptotic nuclei seen in various tissues as well as in mammalian preimplantation embryos (Mohr and Trounson, 1982, Potten etal., 1987). That is why we supposed that nuclear abnormalities observed by us were related to apoptosis. To verify this hypothesis, chromatin distribution and DNA breakage were investigated in a group of embryos with preserved nuclear and cell membranes fixed in paraformaldehyde. The presence 


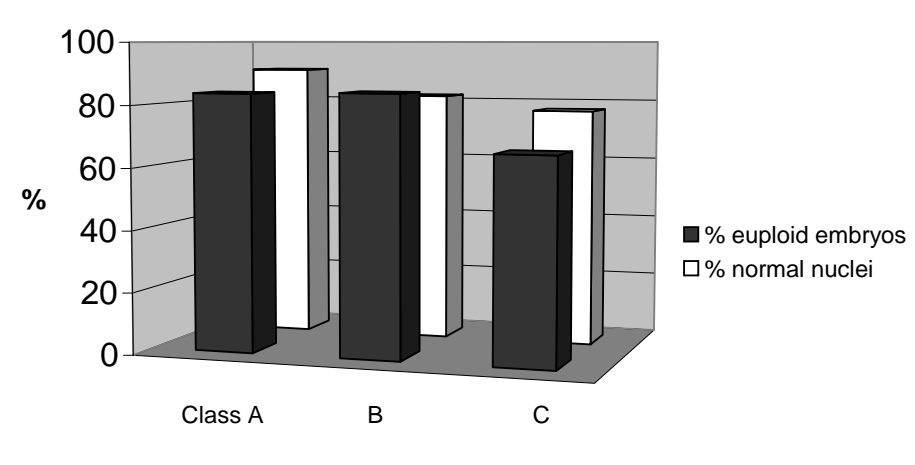

of apoptotic process was confirmed by nuclear morphology and positive TUNEL. Nearly the same proportions of normal and abnormal nuclei were registered by TUNEL and chromatin fluorescent reaction. These facts support our previous assumption that in human preimplantation embryos nuclear lesions tend to precede cellular fragmentation and nuclear apoptosis can be seen in a substantial portion on embryos with good morphology (Delimitreva, 2002). Discussing the condensation of the chromatin as a sign of nuclear apoptosis, the asynchronous division in mammalian embryos should be taken into account - in some neighbour blastomeres unequal chromatin condensation could be a result of initial chromosomal condensation in early prophase. In addition, asynchrony of mitotic cycles of the blastomeres may lead to doubtful interpretation of double or split FISH signals in interphase nuclei before and after DNA replication (Pujol et al., 2004).

In this study, dying nuclei were observed in nearly $1 / 10$ of blastomeres of good embryos (class A and B) and more than 1/ 5 of class $C$ embryos. The chromatin in these nuclei shows apoptotic lesions. This was supported by images of fragmented and/or condensed nuclei and the positive TUNEL-test in apparently normal nuclei. Our results are in accordance with the hypothesis that in preimplantation embryos cytoplasmic and nuclear destruction are relatively independent and the links between them differ from those in the later developmental stages. It is well known that cell cycle regulation in preimplantation embryos is very specific - the start of mitosis is not related to cytoplasmic growth and the checkpoints are not effective. In the damaged cells, apoptotic self-destruction is an alternative of mitosis and is controlled by the general cell cycle mechanisms. The relative independence of nuclear apoptosis from the cell fragmentation observed in our study represents additional evidence for the oddity of the cell cycle in preimplantation embryos. The percentage of chromosomally normal embryos was calculated by two approaches: as a portion of successfully tested embryos (termed ploid normality) and as a portion of all tested embryos (total normality). In the first case, embryos with a substantial proportion of damaged nuclei that precludes reliable FISH were excluded from the calculations. In the second case, embryos with damaged nuclei were included in the group of abnormal embryos. Both total and ploid normality were very similar for class A and B embryos. That means that minimal blastomere fragmentation is not related to increase of chromosomal abnormality. Ploid normality was slightly lower for class $C$ embryos in relation to class $A$ and $B$. The difference was considerably higher for the total normality. The most likely explanation is that in the group of embryos with poor

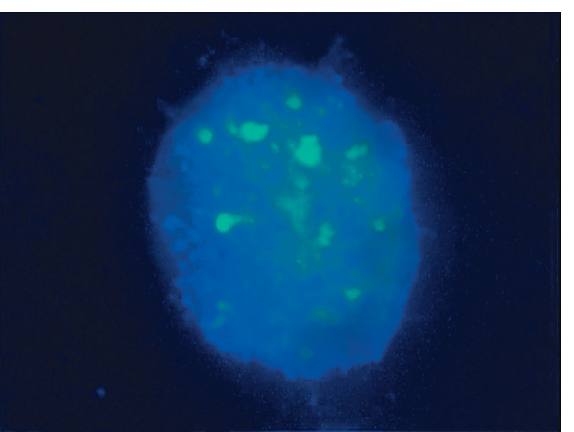

Fig. 4 (Left). Morphology vs. Ploid and nuclear normality. Distribution of blastomeres with abnormal chromatin and embryos with abnormal ploidy in morphological classes $A, B$ and $C$.

Fig. 5 (Right). TUNEL. $A n$ apparentlynormal nucleus with positive TUNEL reaction.

morphology, a considerable portion of nuclei with abnormal ploidy start apoptosis, their ploid status cannot be defined and part of chromosomally abnormal embryos remains untested. The embryos involved in this study were tested for four chromosomes part of them for X-chromosome and a set of autosomes $(1,5,19)$, which are not frequently involved in late fetal loss. The rest of the embryos were tested for chromosomes 18, 21, $\mathrm{X}$ and $\mathrm{Y}$. It is known that aneuploid combinations of these chromosomes are the major part of karyotype abnormalities in newborns. The rates of ploid normality in both groups were close - $83 \%$ and $86.1 \%$ respectively. As we expected, the percentage of chromosomally normal embryos registered by us was higher than reported in the studies investigating 7 - 10 chromosomes (Gianaroli et al., 1999, Marques et al., 2000, Rubio et al., 2003, Staessen et al., 2004). We must have in view three groups of factors, contributing to the relatively high rate of ploid normality - the selection of the patients, exclusion of arrested embryos and exclusion of blastomeres with condensed, flocculent and fragmented nuclei (regardless of the presence and number of FISH signals).

Most of the studies investigating the rate of chromosome abnormalities at cleavage stage are based on analysis of embryos derived form patients with pour prognosis to achieve pregnancy (advanced maternal age, multiple implantation failures, recurrent spontaneous abortions), arrested embryos are also included. In our study only couples with good prognosis were involved - female patients were between 23 and 38 years old, at least 5 mature oocytes were aspirated and at least $4 / 5$ of the oocytes were fertilized successfully. Nuclear abnormalities in blastomeres are related to defects of mitotic spindle and cytokinesis (Chatzimeletiou et al., 2005, Kligman et al., 1996). It is known

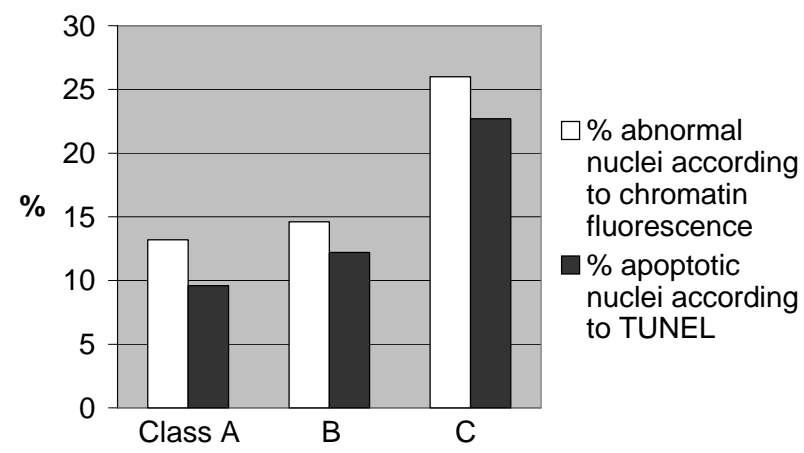

Fig. 6. Morphology / Apoptotic rate. Percentage of abnormal nuclei according to TUNEL and fluorescent chromatin reaction in morphological classes $A, B$ and $C$. 
that the rate of ploid errors is very high in abnormal blastomere nuclei. According to our observation of the chromatin status, a substantial part of abnormal nuclei display morphological features of apoptotic destruction and hybridization in them has partial success. On the other hand, apoptotic blastomeres can not cleave and their genetic defects could not be inherited in the in the next cleavage stages. These are the reasons to analyse FISH results only in normal interphase and mitotic chromatin. We think that exclusion of fragmented, flocculent and condensed nuclei is related to the low rate of chromosomal abnormalities registered by us. There are some attempts to select embryos after PGD for up to ten chromosomes (Bielanska et al., 2002, Munne et al., 1999, Munne, 2003). It was supposed that the replacement of selected embryos would improve considerably the success rate of IVF procedures. Two conclusions were obtained-first, the increase of implantation rate was not significant and second, slight decrease of embryo loss after implantation was achieved (Munne et al., 1999). This could be explained by the following facts - first, only one or two blastomeres per embryo are tested in PGD and the conclusion could be wrong because of mosaicism; and second, probably even serious disturbances of the embryonic chromosomal set are compatible with early pregnancy (Delhanty, 2001). Investigations of all chromosomes in all blastomeres of nontransferred embryos by comparative genome hybridization (CGH) revealed that $1 / 4$ of human cleaving embryos contain only chromosomally normal blastomeres (Wells and Delhanty, 2000). CGH is not suitable for routine PGD so far, because it requires prolonged in vitro culture of the embryos. Besides, it does not solve the problem with mosaicism. In general, the potential of preimplantation chromosomal testing for selection of most viable embryos (apart from cases with known family problems) remains questionable and this approach is applied in a very limited number of IVF centers (Munne, 2003). In most fertility clinics, like ten years earlier, the selection of replaced embryos is based on their morphology (Alikani et al., 2000, Giorgetti et al., 1995, Hoover et al., 1995). In our IVF center the practice is the same. It is to a certain extent hazardous approach.

In this study we have investigated embryos from patients with good prognosis to achieve pregnancy. No difference was noted in the proportions of morphological, chromosomal and chromatin abnormalities for embryos donated after successful and unsuccessful IVF cycles. That means that supernumerary embryos from unsuccessful procedures (at least these with relatively good morphology) are adequate source of data for studying the contribution of chromosomal disorders, nuclear and cell destruction to the early embryonic loss.

In conclusion, our results showed that the minimal cell fragmentation is not related to increase of the proportions of blastomeres with abnormal chromosomal and chromatin status. Our detailed observation of chromatin distribution in all fixed blastomeres showed that the conventional microscopic estimation of nuclear normality can be inadequate. This was illustrated by fluorograms of consequent stages of nuclear destruction in human cleavage stage embryos. Apoptotic chromatin lesions are the only limiting factor for the success of FISH investigation. Nearly 1/2 of embryos with at least one apoptotic nucleus were chromosomally abnormal. For the embryos that contain only normal nuclei the rate of ploid normality was close to $9 / 10$. The rate of euploidy was higher $(66 \%)$ in embryos with significant degree of fragmentation. In a substan- tial portion of abnormal blastomeres chromatin damages precede cell fragmentation. Although the aim of this work was not development of an embryo selection approach, our results could contribute to specification of criteria for new non-invasive embryo classification and selection systems, which are being introduced in several laboratories (De Plachido et al., 2002, Hardarson et al., 2001, Moriwaki et al., 2004). In addition to the practical benefit for IVF treatment, investigations of the correlations between blastomere fragmentation, nuclear destruction and cell cycle disturbances could be useful for the understanding of the causes and mechanisms underlying the developmental arrest of human preimplantation embryos.

\section{Materials and Methods}

This study was approved by the Ethical Committee of Preclinical University Center - Medical University of Sofia and the patients gave informed consent for the supernumerary embryos to be used for scientific research. The investigated embryos were donated for research purposes in the cases when more than the required number of embryos with good morphology and adequate mitotic rate were available on the day of embryo transfer. Only couples with good prognosis for achieving pregnancy were involved in our study. The mean maternal age was 33 years (range 23 - 38). In order to prevent involvement of unknown prezygotic factors, which may decrease the chance for pregnancy, patients with less than five mature oocytes and fertilization rate less than $4 / 5$ were excluded from the study.

\section{Ovarian stimulation}

The IVF and embryo transfer procedures were carried out according to the protocols applied in the Human IVF Unit based at the Department of Biology, Medical Faculty of Sofia (Vatev, 1988, 1992, Vatev et al., 1998). Pituitary down-regulation was achieved by treatment with gonadotrophinreleasing hormone analogue (Decapeptyl, Ferring-Leciva, Czech Republic or Synarel, Syntex Laboratories, USA). Ovarian stimulation was induced using human menopausal gonadotrophin (Humegon, Organon, Holland or Pergonal, Serono, Italy) or recombinant follicle stimulating hormone (Puregon, Organon). Transvaginal follicular aspiration was performed 36 hours after human chorionic gonadotrophin injection (Pregnyl, Organon or Profasi, Serono). Embryos were cultured in Medicult culture media (MediCult, Denmark) or ASP-100 medium (Scandinavian IVF Science AB, Sweden).

\section{Morphology evaluation}

A total of 211 normally fertilized embryos (originated from two-pronuclear zygotes) were included in this study. The embryos in which cell division was not observed for the last 24 hours before fixation were determined as arrested and excluded from the study. The cleaving embryos were fixed $50-52$ or $74-76$ hours post insemination, respectively at 3-7 cell stage and 6-10 cell stage. The presence of cell fragments was examined before the fixation. According to the rate of blastomere fragmentation, embryos were subdivided into three morphological classes - class $A$ (without any blastomere fragments), class $B$ (cell fragments occupied up to $20 \%$ of intrazonal space), class C (cell fragments occupied up to $50 \%$ of intrazonal space). Embryos with apparently necrotic cells, non-translucent cytoplasm, unclear cell borders and more than $50 \%$ fragmentation were excluded from the study. Two-cell stage embryos were also excluded, because a substantial part of them were classified as arrested.

\section{Ploidy evaluation}

A hundred and eighty-three embryos were prepared for fluorescent in situ hybridization (FISH). They were tested for four chromosomes in two FISH rounds. Zonae pellucidae were removed by Tyrode solution, $\mathrm{pH} 2.5$, adjusted by $\mathrm{HCl}$. The embryos were incubated for $5 \mathrm{~min}$ at room temperature in hypotonic solution (1\% sodium citrate with $5 \%$ BSA to prevent 
adhesion to the dish), dropped in minimal volume onto immediately cleaned by acetone slides and fixed by freshly mixed methanol/acetic acid (3:1). Fixation was controlled under phase-contrast microscope. Fixative was dropped and dried till the complete disappearance of the cytoplasm. The place of fixed blastomeres was carefully marked by diamond pencil. The slides were kept at -20 ...C until the FISH testing. Following FISH probes were used: $\alpha$-satellite DNA specific for centromeric regions of chromosomes 1, 5, 19 and X, FITC labeled (Boehringer Mannheim, Austria), CEP 18 Spectrum Green, for the centromeric region of chromosome 18 (Vysis, Germany), LSI21 Spectrum Orange - a locus specific probe for the long arm of chromosome 21 (Vysis), centromeric probes for $\mathrm{X}$ chromosome, FITC labeled (Q-biogene, Germany), CEP X Spectrum Green (Vysis) and $Y$ chromosome classical satellite probe, Texas Red labeled (Q-biogene).

The embryos prepared for FISH were divided into two groups. A group of 52 embryos was tested for chromosomes 1, 5, 19 in the first FISH round and for $X$ chromosome in the second one. The remaining group of 131 embryos was tested for chromosomes 18 and 21 in the first FISH round and for $X$ and $Y$ in the second one. Between the two rounds slides were immersed for 10 seconds in distilled water at 71 ...C to remove hybridized probes, dried at $60 \ldots \mathrm{C}$ for an hour and dehydrated in ethanol $(70,85,100 \%)$. We used commercially obtained hybridization mixtures for Vysis and Q-biogene probes (for chromosomes 18, 21, $\mathrm{X}$ and $\mathrm{Y}$ ) and "in-house made" mixture for BoehringerMannheim probes $(1,5,19$ and $\mathrm{X}$ ). It contained $60 \%$ formamide, $10 \%$ dextran sulfate $500000,2 \mathrm{mg} / \mathrm{ml}$ sonicated salmon sperm DNA (Sigma), $0.1 \mathrm{mg} / \mathrm{ml}$ human Cot1 DNA (Boehringer Mannheim) and directly labeled hybridization probe in final concentration 1-2 $\mathrm{ng} / \mathrm{\mu l}$ (for each chromosome) diluted in 2XSSC, $\mathrm{pH} 7$. The same FISH protocol was used for both commercial and home-prepared mixtures and for both FISH rounds. A drop of $3.5 \mu$ hybridization mixture per embryo was applied under $10 \times 10 \mathrm{~mm}$ coverslips. Nuclear DNA and probes were denatured simultaneously for 3 minutes at $75 \ldots$... and hybridized for 4 hours at $37 \ldots C$ in a humidified chamber. FISH results were examined using Olympus BX60 microscope (Olympus, Germany) equipped with digital camera (Olympus, VC45) and software from MetaSystems (ISIS, MetaSystems, Germany).

Simultaneously with the scoring of FISH signals, the appearance of the chromatin of each nucleus was evaluated. Chromatin status of observed nuclei was defined as normal or abnormal according to the presence of nuclear fragmentation and/or uneven chromatin condensation.

\section{Evaluation of DNA breakage}

In order to evaluate the presence of early apoptotic changes in embryos with different rate of cell fragmentation, a group of 28 randomly chosen embryos from all morphological classes were subjected to terminal deoxynucleotidyl transferase-mediated dUTP nick endlabeling (TUNEL). Zonae pellucidae were removed by acidified Tyrode solution and embryos were immediately fixed for 10 minutes on clean slides in freshly prepared $4 \%$ paraformaldehyde in $\mathrm{PBS} \mathrm{pH}$ 7.3. After drying, slides were stored at $-20 \ldots$..C until further use. All reagents and the manual for TUNEL procedure were provided by R\&D Systems (TACS TdT In Situ Apoptosis Detection Kit, R\&D Systems Europe, UK). Briefly, previously fixed embryos were dehydrated in ethanol series and air dried at 50...C for at least one hour, permeabilized in Cytonin (commercially provided solution) for 15-20 minutes and subjected to terminal transferase reaction with biotinylated nucleotides for an hour at $37 \ldots \mathrm{C}$ in moist chamber. After washing in stop-buffer (provided ready for use) and PBS, labeled DNA nicks were visualized with streptavidin-fluorescein conjugate. The nuclei were counterstained with $125 \mathrm{ng} / \mathrm{ml}$ DAPI (4,6-diamidino-2-phenylindole, Sigma, USA) and $3 \%$ DABCO (diazibicyclo[2.2.2]octane, Sigma) diluted in glycerol/PBS $(9: 1)$ and examined by fluorescent microscopy on Olympus BX60 microscope.

\section{Statistical method}

Statistical analysis was carried out using $\chi^{2}$-test. Differences were considered significant when $\mathrm{P}\left(\chi^{2}\right)<0.05$.

\section{Acknowledgement}

This work was supported by a grant from Medical University, Sofia, project N 22/2002. We thank Dr R. Borissov for the statistical calculations.

\section{References}

ALIKANI, M., CALDERON, G., TOMKIN, G., GARRISI, J., KOKOT, M. and COHEN, J. (2000) Cleavage anomalies in early human embryos and survival after prolonged culture in vitro. Hum. Reprod. 14: 2634-2643.

ANTCZAK, M. and VAN BLERKOM, J. (1999) Temporal and spatial aspects of fragmentation in early human embryos: possible effects on developmental competence and association with the differential elimination of regulatory proteins from polarized domains. Hum. Reprod. 14: 429-447.

BALAKIER, H. and CADESKY, K. (1997) The frequency and developmental capability of human embryos containing multinucleated blastomeres. Hum. Reprod. 12: 800-804.

BIELANSKA, M., TAN, S.L. and AO, A. (2002) Chromosomal mosaicism throughout human preimplantation development in vitro: incidence, type and relevance to embryo outcome. Hum. Reprod. 17: 413-419.

BUSTER, J.E., BUSILLO, M., RODI, I.A., COHEN, S.W., HAMILTON, M., SIMON, J.A., THORNEYCROFT, I.H. and MARSHALL, J.R. (1985) Biologic and morphologic development of donated human ova recovered by nonsurgical uterine lavage. Am. J. Obstet. Gynecol. 153: 211-217.

CHATZIMELETIOU, K., MORRISON, E.E., PRAPAS, N., PRAPAS, Y. and HANDYSIDE, A.H. (2005) Spindle abnormalities in normally developing and arrested human preimplantation embryos in vitro identified by confocal laser scanning microscopy. Hum. Reprod. 20(3):672-682.

COONEN, E.J., HARPER, C., RAMAEKERS, F.C.S., DELHANTY, J.D., HOPMAN, A.H, GERAEDTS, JP and HANDYSIDE, A.H. (1994) Presence of chromosomal mosaicism in abnormal preimplantation embryos detected by FISH. Hum. Genet. 94: 609-615.

DE PLACIDO, G., MARTIN, W., STRINA, I., ALVIGGI, E., ALVIGGI, C., MOLLO, A., VARICCHIO, M.T., TOLINO, A., SCHIATTARELLA, C. and DALE, B. (2002) High outcome predictability after IVF using a combined score for zygote and embryo morphology and growth rate. Hum. Reprod. 17: 2402-2409.

DELHANTY, J.D. (2001) Preimplantation genetics: an explanation for poor human fertility? Ann. Hum. Genet. 65: 331-338.

DELIMITREVA, S., ZHIVKOVA, R. and VATEV, I. (2001) Supernumerary human preembryos provide potential for preimplantation genetic diagnosis. Folia Biol. (Praha) 47: 88-91.

DELIMITREVA, S.M. (2002) The status of the chromatin of human preimplantation embryos with good morphology. Folia Biol. (Praha) 48: 149-153.

EVSIKOV, S. and VERLINSK,Y.Y. (1998) Mosaicism in the inner cell mass of human blastocysts. Hum. Reprod. 13: 3151-3155.

FULKA, J. Jr., FIRST, N.L., FULKA, J. and MOOR, R.M. (1999) Checkpoint control of the G2/M phase transition during the first mitotic cycle in mammalian eggs. Hum. Reprod. 14: 1582-1587.

GIANAROLI, L., MAGLI, M.C., MUNNE, S., FORTINI, D. and FERRARETTI, A.P (1999) Advantages of day 4 embryo transfer in patients undergoing preimplantation genetic diagnosis of aneuploidy. J. Assist. Reprod. Genet. Apr;16(4):1705.

GIORGETTI, C., TERROU, P. and AUQUIER, P. (1995) Embryo score to predict implantation after in vitro fertilization: based on 957 single embryo transfers. Hum. Reprod. 10: 1427-2431.

HANDYSIDE, A.H. (1996) Mosaicism in the human preimplantation embryo. Reprod. Nutr. Dev. 36: 643-649.

HARDARSON, T., HANSON, C., SJOGREN, A. and LUNDIN, K. (2001) Human embryos with unevenly sized blastomeres have lower pregnancy and implantation rate: indications for aneuploidy and multinucleation. Hum. Reprod. 16: 313318. 
HARDY, K., WINSTON, R.M.L. and HANDYSIDE, A.H. (1993) Binucleate blastomeres in preimplantation in preimplantation human embryos in vitro: failure of cytokinesis during early development. J. Reprod. Fertil. 98: 549-558.

HARDY. K., WRIGHT, C., RICE, S., TACHATAKI, M., ROBERTS, R., MORGAN, D., SPANOS, S. and TAYLOR, D. (2002) Future developments in assisted reproduction in humans. Reproduction. 123: 171-183.

HOOVER, L., BAKER, A., CHECK, J.H., LURIE, D. and O'SHAUGHENESSY, A. (1995) Evaluation of a new embryo-grading system to predict pregnancy rates following in vitro fertilization. Gynecol. Obstet. Invest. 40: 151-157.

HUNT, P.A. and HASSOLD, T.J. (2002) Sex matters in meiosis. Science. 296 2181-2183.

JURISICOVA, A., VARMUZA, S. and CASPER, R.F. (1996) Programmed cell death and embryo fragmentation. Mol. Hum. Reprod. 2: 93-98.

KLIGMAN, I., BENADIVA, C., ALIKANI, M. and MUNNE, S. (1996) The presence of multinucleated blastomeres in human embryos is correlated with chromosomal abnormalities. Hum. Reprod. 11: 1492-1498.

MAGLI, M.C., JONES, G.M., GRAS, L., GIANAROLLI, L., KORMAN, I. and TROUNSON, A.O. (2000) Chromosome mosaicism in day 3 aneuploid embryos that develop to morphologically normal blastocyst in vitro. Hum. Reprod. 15, 2000: 1781-1786.

MARQUEZ, C., SANDALINAS, M., BAHCE, M., ALIKANI, M. and MUNNE, S. (2000) Chromosome abnormalities in 1255 cleavage-stage human embryos. Reprod. Biomed. Online.1(1):17-26.

MARTINEZ, F., RIENZI, L., IACOBELLI, M., UBALDI, F., MENDOZA, C., GRECO, E. and TESARIK, J. (2002) Caspase activity in preimplantation human embryos is not associated with apoptosis. Hum. Reprod. 17: 1584-1590.

MOHR, R.L. and TROUNSON, A.O. (1082) Comparative ultra structure of hatched human, mouse and bovine blastocysts. J. Reprod. Fertil. 66: 506-509.

MORIWAKI, T., SUGANUMA, N., HAYAKAWA, M., HIBI, H., KATSUMATA, Y., OGUCHI, H. and FURUHASHI, M. (2004) Embryo evaluation by analysing blastomere nuclei. Hum. Reprod. 19: 152-156.

MUNNE, S., ALIKANI, M., TOMKIN, G., GRIFO, J. and COHEN, J. (1995) Embryo morphology, developmental rates and maternal age are correlated with chromosome abnormalities. Fertil. Steril. 64:382-91.

MUNNE, S., MARQUEZ, C., MAGLI, C., MORTON, P. and MORRISON, L. (1998) Scoring criteria for preimplantation genetic diagnosis of numerical abnormalities for chromosomes X, Y, 13, 16, 18 and 21. Mol. Hum. Reprod. 4: 863-70.

MUNNE, S., MAGLI, C., COHEN, J., MORTON, P., SADOWY, S., GIANAROLI, L., TUCKER, M., MARQUEZ, C., SABLE, D., FERRARETTI, A.P., MASSEY, J.B. and SCOTT, R. (1999) Positive outcome after preimplantation diagnosis of aneuploidy in human embryos. Hum. Reprod. 14: 2191-2199.

MUNNE, S. (2003) Preimplantation genetic diagnosis and human implantation. A review. Placenta, 24, 1-7.

PEllestor, F., GIRARDET, A. ANDREO, B., ARNAL, F. AND HUMEAU, C.
(1994) Relationship between morphology and chromosomal constitution in human preimplantation embryo. Molec. Reprod. Dev. 39: 141-146.

PICKERING, S.J., TAYLOR, A., JOHNSON, M.H. and BRAUDE, P.R. (1995) An analysis of multinucleated blastomere formation in human embryos. Hum. Reprod. 10: 1912-1922.

POTTEN, C.S., ed., KERR, J.F.R., SEARLE, J., HARMON, B.V. and BISHOP, C.J. (1987) Perspectives on mammalian cell death. Apoptosis. pp 93-128, Oxford, Oxford University Press.

PUJOL, A., BENET, J., CAMPILLO, M., CODINA-PASCUAL, M., EGOZCUE, J. and NAVARRO, J. (2004) The use of a cell-cycle phase-marker may decrease the percentage of errors when using FISH in PGD. Cytogenet. Genome. Res. 105(1):29-35.

RUBIO, C., SIMON, C., VIDAL, F., RODRIGO, L., PEHLIVAN, T., REMOHI, J. and PELLICER, A. (2003) Chromosomal abnormalities and embryo development in recurrent miscarriage couples. Hum. Reprod. Jan;18(1):182-8.

StAESSEN, C., PLATTEAU, P., VAN ASSCHE, E., MICHIELS, A., TOURNAYE, H., CAMUS, M., DEVROEY, P., LIEBAERS, I. AND VAN STEIRTEGHEM, A. (2004) Comparison of blastocyst transfer with or without preimplantation genetic diagnosis for aneuploidy screening in couples with advanced maternal age: a prospective randomized controlled trial. Hum. Reprod. 19(12):2849-58

VAN BLERKOM, J., DAVIS, P. and ALEXANDER, S. (2001) A microscopic and biochemical study of fragmentation phenotypes in stage-appropriate human embryos. Hum. Reprod. 16: 719-729.

VAN ROYEN, E., MANGELSCHOTS, K., VERCRUYSSEN, M., DE NEUBOUR,G. D., VALKENBURG, M., RYCKAERT, G. and GERRIS, J. (2003) Multinucleation in cleavage stage embryos. Hum. Reprod. 18: 1062-1069.

VATEV, I. (1998) Human in vitro fertilization and embryo transfer program at the Medical Academy, Sofia, Bulgaria. J. In Vitro Fert. Embryo Transfer. 5: 48-49.

VATEV, I,TS. (1992) Development of IVF technology in Bulgaria. ARTA 3: 135-136.

VATEV, I., KARAGYOZOV, I., ISTATKOV, M. and SREBREVA, M. (1998) Successful application of the fallopian sperm perfusion method and intracytoplasmic sperm injection (ICSI) technique for treatment of human infertility. Balkan J. Med. Genet. 3: 115-119.

WARNER, C.M., CAO, W., EXLEY, G.E., MCELHINNY, A.S., ALIKANI, M., COHEN, J., SCOTT, R.T. and BRENNER, C.A. (1998) Genetic regulation of egg and embryo survival. Hum. Reprod. 13: 178-196.

WELLS, D. and DELHANTY, J.D. (2000) Comprehensive chromosomal analysis of human preimplantation embryos using whole genome amplification and single cell comparative genomic hybridization. Mol. Hum. Reprod. 6: 1055-1062.

Received: October 2004

Reviewed by Referees: February 2005

Modified by Authors and Accepted for Publication: April 2005 